\title{
Planets in binary systems: is the present configuration indicative of the formation process?
}

\author{
F. Marzari ${ }^{1}$ and M. Barbieri ${ }^{2}$ \\ 1 Dipartimento di Fisica, University of Padova, via Marzolo 8, 35131 Padova, Italy \\ e-mail: marzari@pd.infn.it \\ 2 LAM, Traverse du Siphon, BP 8, Les Trois Luc, 13376 Marseille Cedex 12, France \\ e-mail: mauro.barbieri@oamp.fr
}

Received 15 January 2007 / Accepted 22 January 2007

\begin{abstract}
Aims. The present dynamical configuration of planets in binary star systems may not reflect their formation process since the binary orbit may have changed in the past after the planet formation process was completed. An observed binary system may have been part of a former hierarchical triple that became unstable after the planets completed their growth around the primary star. Alternatively, in a dense stellar environment even a single stellar encounter between the star pair and a singleton may singificantly alter the binary orbit. In both cases the planets we observe at present would have formed when the dynamical environment was different from the presently observed one.

Methods. We have numerically integrated the trajectories of the stars (binary plus singleton) and of test planets to investigate the abovementioned mechanisms. The orbits of the planets have been computed to test the survival of a planetary system around the primary during the chaotic phase of the stars.

Results. Our simulations show that the circumstellar environment during planetary formation around the primary was gravitationally less perturbed when the binary was part of a hierarchical triple because the binary was necessarely wider and, possibly, less eccentric. This circumstance has consequences for the planetary system in terms of orbital spacing, eccentricity, and mass of the individual planets. Even in the case of a single stellar encounter the present appearance of a planetary system in a binary may significantly differ from what it had while planet formation was ongoing. However, while in the case of instability of a triple the trend is always towards a tighter and more eccentric binary system, when a single stellar encounter affects the system the orbit of the binary can become wider and be circularized.

Conclusions. Modelling the formation of a planetary system around a binary is a potentially complex task and an effort has to be made to look into its present dynamics for traces of a possible chaotic past.
\end{abstract}

Key words. planets and satellites: formation - methods: $N$-body simulations - celestial mechanics stars: planetary systems: formation - stars: planetary systems

\section{Introduction}

Among the many extrasolar planets discovered so far, more than 40 are gas giants detected in binary or even triple star systems. This is not an unexpected result since it is known that about half of solar type stars near the sun have a companion and there is evidence that the binary frequency might be higher among young stars (Reipurth \& Zinnecker 1993; Kohler \& Leinert 1998; Ghez et al. 1993). Most of the binary systems hosting a planet have a wide separation ranging from 100 to $1000 \mathrm{AU}$ (see Desidera \& Barbieri 2007, for a compilation of binary systems with planets). However, there are some systems like $\gamma$ Cephei, Gl 86 and HD 41004A where the separation between the two stars is of the order of $20 \mathrm{AU}$ or less. Planetary formation in these systems is perturbed to a significnat extent by the gravitational influence of the companion star possibly altering the process. Thébault et al. (2004) have shown that the formation of the planet in $\gamma$ Cephei via core-accretion (Pollack et al. 1996) is unclear. The migration in the massive gaseous disk required to form a planet within 2-2.5 AU from the star leads to an orbit much closer to the star than that observed. On the other hand, Boss (2006) has shown that in hydrodynamical models with artificial viscosity the presence of a companion star may help to trigger the formation of giant planets by disk instability.

The different models for planetary formation are applied considering that the present orbital configuration of the system star-planet(s)-star was unchanged. However, there are cases where the orbit of the companion star may have been modified between the time when planetary formation was ongoing and the present. If the binary separation was wider or the eccentricity lower, the protoplanetary disk would have been a more quiet environment promoting the formation of planets. There are two possible mechanisms that may have altered a past binary dynamical configuration into the presently observed one.

- We assume that the observed binary system was in the past part of an unstable larger multiple system (triple or more). We will concentrate here on hierarchical triples, the most common multiple system, in which the inner binary is orbited by a third star on a wider orbit. The dynamical evolution of these systems may be complex in particular in the presence of high inclinations between the binary and the singleton. We may have systems whose parameters change nearly periodically by large amounts that are stable over long timescales. However, if the triple is dynamically unstable 
and the system becomes chaotic, the most frequent outcome (stellar collisions are rarer) is the ejection of a star leaving a binary system with smaller separation, because of orbital energy conservation, and higher eccentricity. The planet in a circumstellar orbit around the main star of the pair has a time window to form in a quiet disk before the onset of dynamical instability of the triple system and subsequent modification of the original binary orbit into a more eccentric and closer one.

- If the binary system formed in a densely populated cluster it is likely that the star couple underwent an encounter with another star. During the close stellar passage the orbital parameters of the binary system are permanently modified leading to a different configuration star-planet(s)-star. Even in this case the mean trend is towards more eccentric orbits and, on average, to an orbital shrinking of the binary pair (Heggie 1975; Larson 2001).

Both these mechanims, besides altering the binary configuration, have also the potential to destabilize the circumprimary orbits of potential planets. However, as we will see in the following, inner circumprimary orbits, like that of the planet in $\gamma$ Cephei, are little or not affected by the stellar encounters in most cases.

In Sect. 2 of this paper we will describe how the decay of a hierarchical triple star system affects the orbital parameters of the binary system that was part of it and of a putative planetary system around the primary star of the system. To simplify the approach we will model only coplanar hierarchical systems even if inclined triples may be more common because of frequent encounters in star forming regions early on in stellar evolution. This restriction to coplanarity is suggested by the minor complexity of the star dynamics, compared to the high inclined systems, that allows us to ouline clearly the effects of the stellar instability on the planet environment. We will also investigate to what extent the close encounters between the secondary stars perturb a planetary system around the main star. In Sect. 3 we will analyse the effects of stellar encounters on a binary system with planets. We will present selected cases that illustrate the difficulty of interpreting the formation of a planet in a binary system in light of its present orbital paramters. We will not perform a full exploration of the parameter space for the two mechanisms described in Sects. 2 and 3 which would be beyond of the scope of this paper.

\section{Jumping stars and planet survival}

According to Reipurth (2000), Kroupa (1995), and Larson (2001) most binaries and even single stars originate from the decay of multiple star systems. The wide range of orbital parameters observed among binaries might be a consequence of the chaotic dynamics of the primordial multiple systems. Dynamical interactions among the members of the system cause an exchange of energy and angular momentum often resulting in the ejection of a star from the system. This may occur in the early stages of the system evolution or it may take some time for instability to build up. We will consider a scenario in which a triple star system is stable long enough to allow planet formation around the primary star of the binary pair. The subsequent destabilization of the triple, because of the onset of chaotic behaviour, will end with the ejection of one star. The binary couple will have orbital parameters that are at present different from those of the primordial binary in which planetary formation occurred. In some cases, during the stellar encounters an exchange can also occur between the original companion star and the outer one leading to a new mass ratio of the binary system.

\subsection{The numerical algorithm}

To analyse the dynamical evolution of triple star systems and of planetary orbits around the primary star we have assembled a numerical model of the hierarchical three-body system. We define two initial osculating Keplerian orbits for the triple, an inner one for the binary pair $m_{1}$ and $m_{2}$ and an outer one, defined in the center of mass of the pair, for the single star $m_{3}$. Ten additional circular orbits for massless bodies starting at $1 \mathrm{AU}$ from the primary star and extending by equal steps up to $10 \mathrm{AU}$ are also computed. Their evolution allows us to evaluate to what extent the chaotic phase of stellar encounters affects a putative planetary system around the primary star. The evolution of the system is calculated with the numerical integrator RADAU (Everhart 1985) which properly handles close encounters between the massive bodies.

The parameter space of the hierarchical three-body problem is rather wide since in principle there are 12 degrees of freedom: the six parameters of the binary pair and the six ones of the singleton. In this work we restrict ourself to a limited number of significant test cases showing how a detached binary system has its orbital elements changed when the triple is disintegrated and the single star escapes to infinity. A similar behaviour occurs also in non-coplanar systems when the dynamics becomes chaotic, however we will perform a full exploration of the inclined case in a forthcoming paper. We start with the stars in prograde and coplanar orbits with the semimajor axis of the binary $a_{\mathrm{b}}$ fixed to $35 \mathrm{AU}$. The semimajor axis of the single star $a_{\mathrm{s}}$ is instead regularly sampled starting within the empirical stability limit derived by Eggleton \& Kiseleva (1995). We consider different values of $e_{\mathrm{b}}$, the eccentricity of the binary, and of $e_{\mathrm{s}}$, the eccentricity of the singleton. Because of the chaotic character of the dynamics, for each set of $\left(a_{\mathrm{b}}, a_{\mathrm{s}}, e_{\mathrm{b}}, e_{\mathrm{s}}\right)$ we perform 30 different simulations with random choices of the orbital angles. In this demonstrative set of simulations the masses of the stars are 1,0.4 and 0.4 solar masses, respectively.

\subsection{The onset of instability}

In Fig. 1 we plot the timescale $t_{\text {in }}$ required for the onset of instability, and then close encounters between the single star and the binary, as a function of the semimajor axis of the singleton. In the first case (red triangles) the initial orbital eccentricities $e_{\mathrm{b}}$ and $e_{\mathrm{s}}$ are both set to 0.2 . There is a trend towards higher values of $t_{\text {in }}$ for larger values of $a_{\mathrm{s}}$ that can be roughly described by an exponential law. Since we stop the simulations after 50 Myr because of too long integration times, the curve is biased at the end of the fit by vales that are all equal to 50 Myr. Despite this limitation of the fit, at a first sight the analytical dynamical stability threshold (Eggleton \& Kiseleva 1995; Kiseleva et al. 1996), located at about $a_{\mathrm{s}}=150 \mathrm{AU}$ for the singleton, appears to be in good agreement with our numerical results if one looks at the fast growing trend of the instability time when $a_{\mathrm{s}}$ is larger than $150 \mathrm{AU}$. In the second case shown in Fig. 1 (blue triangles) we have incresed both the eccentricities $e_{\mathrm{b}}$ and $e_{\mathrm{s}}$ to 0.4 . The instability onset occurs, as expected, faster than the previous case and larger values of $a_{\mathrm{s}}$ are required to have long surviving systems. To find systems whose $t_{\text {in }}$ is longer than $50 \mathrm{Myr}$ we have to go beyond $a_{\mathrm{s}} \sim 250 \mathrm{AU}$. The stability limit set by the formula of Eggleton \& Kiseleva (1995) and Kiseleva et al. (1996) is at 


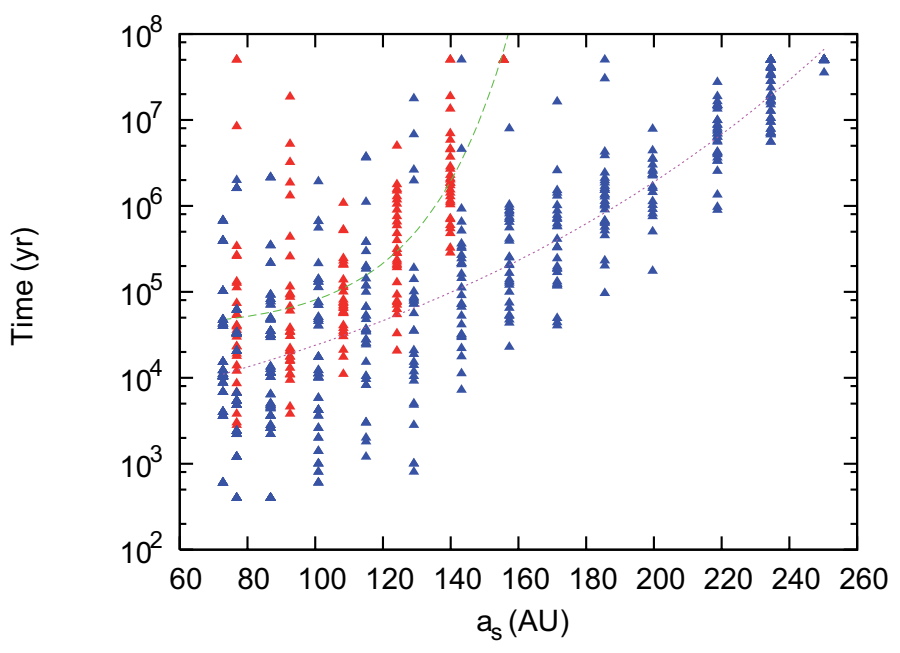

Fig. 1. Time of the first close encounter between the binary and the singleton vs. $a_{\mathrm{s}}$, the initial semimajor axis of the singleton. The red dots mark the cases with binary eccentricity $e_{\mathrm{b}}=0.2$ and singleton eccentricity $e_{\mathrm{s}}=0.2$, the blue dots those with $e_{\mathrm{b}}=0.4$ and $e_{\mathrm{s}}=0.4$. The continuous lines are least squares exponential fits to the data.

about $235 \mathrm{AU}$ and it seems to be slightly less accurate at high eccentricities of the stars.

According to Boss (2006) disk instability can form gas giant planets in a few hundred years. In this scenario the time required by the gravitational perturbations among the stars to build up dynamical instability is not a crucial parameter. When $a_{\mathrm{s}}$ is beyond $70 \mathrm{AU}$ the chaotic behaviour onset occurs after a few thousand years giving enough time for planets to form. Eventually, if the planetary system is chaotic after its formation (Weidenschilling \& Marzari 1996; Marzari \& Weidenschilling 2002; Marzari 2005; Rasio \& Ford 1996) it might have enough time to evolve into a stable state before the star system becomes in turn chaotic. In the core accretion theory (Pollack et al. 1996), the formation of gas giant planets requires a few million years. By inspecting Fig. 1 we expect that only systems in the upper part of the plot are dynamically stable long enough to grant a quiet environment for planets to grow around the primary star. However, even in some cases where the singleton is close to the binary, the dynamics turns chaotic only after some million years.

In Fig. 2 we show the final orbital distribution in the $\left(a_{\mathrm{b}}, e_{\mathrm{b}}\right)$ plane of the left-over binaries. While the initial values of $e_{\mathrm{b}}$ and $e_{\mathrm{S}}$ have a strong influence on the instability time $t_{\text {in }}$, they appear non influential for the final orbital distribution of the binaries. The two different distributions shown in Fig. 2 correspond to $e_{\mathrm{b}}=0.2$ and $e_{\mathrm{s}}=0.2$ (red dots) while the green dots mark systems where both the binary and the singleton are on circular orbits. There is no significant difference between the two distributions that overlap in the $\left(a_{\mathrm{b}}, e_{\mathrm{b}}\right)$ plane. The final semimajor axis $a_{\mathrm{b}}$ is substantially smaller than the initial value $\left(a_{\mathrm{b} 0}=35 \mathrm{AU}\right)$ as expected by orbital energy conservation as one of the stars escapes on a positive energy hyerbolic orbit. The spreading of $a_{\mathrm{b}}$ depends both on the different initial values of $a_{\mathrm{s}}$ and on the amount of energy taken away by the singleton. The final values of $e_{\mathrm{b}}$ are randomly distributed and are concentrated at high eccentricities, in most cases larger than 0.4. The results of additional simulations with sampled values of $e_{\mathrm{s}}$ and $e_{\mathrm{b}}$ show a similar distributions in the final orbital distribution of the binary star system.

In Fig. 3 we map the values of the semimajor axis $a_{\mathrm{p}}$ of the outer planetary orbit that remains stable after the chaotic phase

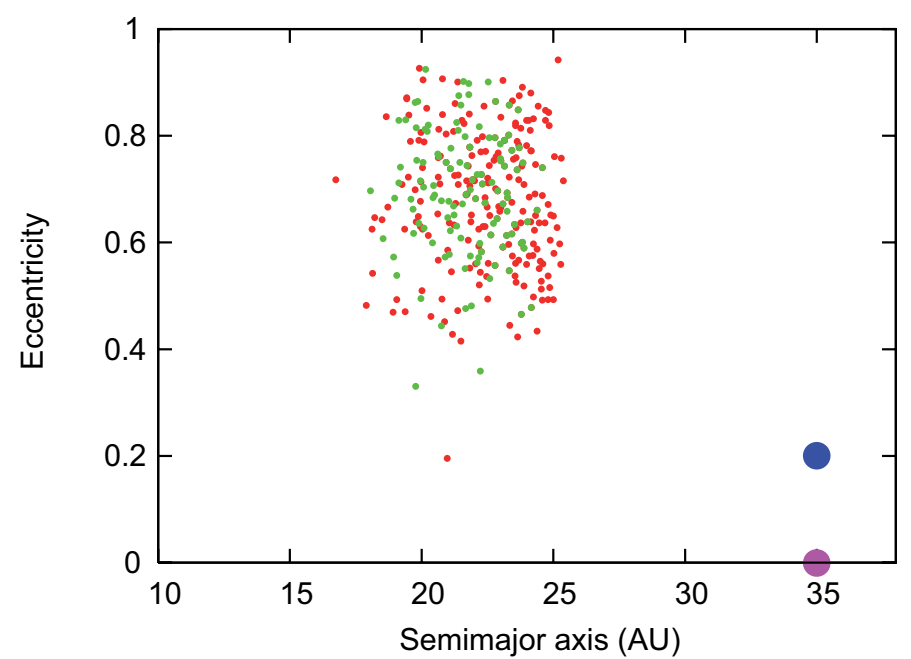

Fig. 2. Distribution of the binary orbital elements after the third star of the system has escaped on a hyperbolic orbit. The red dots label binaries originated from triples with $e_{\mathrm{b}}=0.2$ and $e_{\mathrm{s}}=0.2$, the green dots those from triples with $e_{\mathrm{b}}=0$ and $e_{\mathrm{s}}=0$. The large dots in the plot represent the initial binary system.

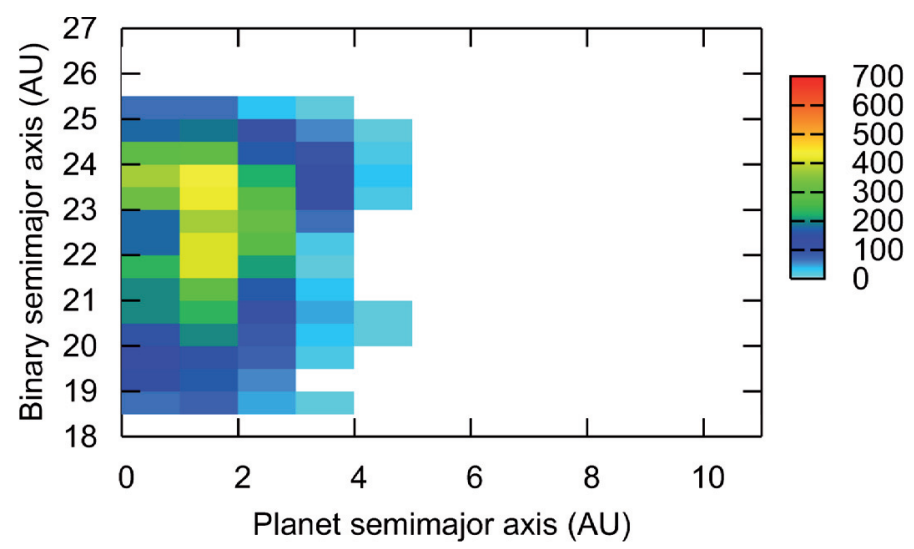

Fig. 3. Histogram illustrating the number of systems in our simulations that, at the end of the chaotic phase, fall into bins in $a_{\mathrm{b}}$, the binary separation, and $a_{\mathrm{p}}$, the semimajor axis of the outer planetary orbit that survives the stellar chaotic phase.

vs. the final value of $a_{\mathrm{b}}$ in the case with $e_{\mathrm{b}}=0.2$ and $e_{\mathrm{s}}=0.2$. The color codes are linked to the number of cases that fall in each bin in $a_{\mathrm{p}}$ ( $x$-axis) and $a_{\mathrm{b}}$ ( $y$-axis). The repeated encounters between the stars set a limit of about 4 AU from the primary star within which planetary orbits can survive. Beyond that value of $a_{\mathrm{p}}$, the gravitational disturbances produced during the chaotic evolution of the two outer stars destabilize any planetary body. It is remarkable that a significant number of binary systems with final values of $a_{\mathrm{b}}$ around $19 \mathrm{AU}$ and high eccentricity, preserve the planetary system, or part of it, that formed when the binary was part of a triple system with $a_{\mathrm{b} 0}=35$ AU. $\gamma$ Cephei, G1 86 and HD 41004A might well belong to this class of systems. In $1 \%$ of our simulations even the innermost planetary orbit we considered ( $\left.a_{\mathrm{p}}=1 \mathrm{AU}\right)$ is perturbed during the stellar chaotic phase and the planet is eventually ejected from the system. This implies that a fraction of planetary systems forming around binaries, part of a triple in the early stages of their evolution, may be totally destabilized during the stellar encounters.

Our study is not exaustive in terms of exploration of the parameter space, however it gives a clear idea of the dynamical evolution of the system. It also suggests caution when 
modelling planetary formation in an observed binary system by simply adopting its present orbital configuration. That configuration might indeed be the outcome of a complex and unpredictable chaotic evolution.

\section{Modification of star-planet-star configurations after a stellar encounter}

Within stellar clusters, close stellar encounters may disrupt binaries or, more frequently, abruptly change their orbital elements (Kroupa 1995). This mechanism is suspected to be one of the possible causes of the low frequency of binaries among lowmass field stars compared to that of young low-mass stars in star-forming associations (Duchene 1999). If a planet or a full planetary system formed around one of the stars of a binary, when a stellar encounter occurs the dynamical configuration of the system may be significantly changed (Dalla Stella 2005). We may see either of the following events:

- the binary system is destroyed and from then on the surviving planets orbit a single star. In this case, the dynamical configuration we observe at present is not indicative of the formation process because of the large changes caused by the stellar encounter;

- the binary system survives the encounter but its orbital parameters and those of the planets are strongly altered.

In both cases, any attempt to model the formation of an observed planetary system would face the problem of discriminating which dynamical or physical features of the system are due to the formation process and which are related to the stellar encounter.

In this section we investigate to what extent the dynamical environment of a planet orbiting a star in a binary system is affected by a stellar encounter that does not disrupt the binary. We adopt the same numerical model described in the previous section, but the singleton is now set on a hyperbolic orbit approaching the binary on a plane tilted by $45^{\circ}$ with respect to the binary orbital plane. We randomly sample the impact parameter and eccentricity of the hyperbolic trajectory and we look at the orbital configuration of the final system star-planet-star when the encounter is over. In Fig. 4 we show the orbital distribution in the $(\mathrm{a}, \mathrm{e})$ and $(\mathrm{a}, \mathrm{i})$ planes of the binary systems after the stellar encounter. A significant number of systems has a postencounter semimajor axis significantly smaller than the initial one $\left(a_{\mathrm{b} 0}=35 \mathrm{AU}\right)$ while during the decay of a triple system the final $a_{\mathrm{b}}$ cannot be lower than a value fixed by the conservation of the gravitational energy of the initially bounded system.

The impact of a stellar encounter on planetary formation models is important: the planet(s) may have formed when the companion star was farther away and then less effective in perturbing the protoplanetary disk. For the core-accretion model (Pollack et al. 1996), this may be a crucial condition to allow planetesimal accumulation before the onset of strong binary perturbations when the companion stars is injected on a closer orbit (Thébault et al. 2004, 2006). By inspecting the planetary orbits that are still bound to the primary star after the encounter shown in Fig. 5, we observe a distribution very different from that of Fig. 3. We still have cases where planetary orbits up to $3 \mathrm{AU}$ survive the encounter leaving the planets around a closer binary system than the initial one. When we look at $\gamma$ Cephei, Gl 86 and HD 41004A we may be observing a system that survived a close stellar encounter. However, it appears that the single encounter with a star on a hyperbolic orbit has less dramatic consequences
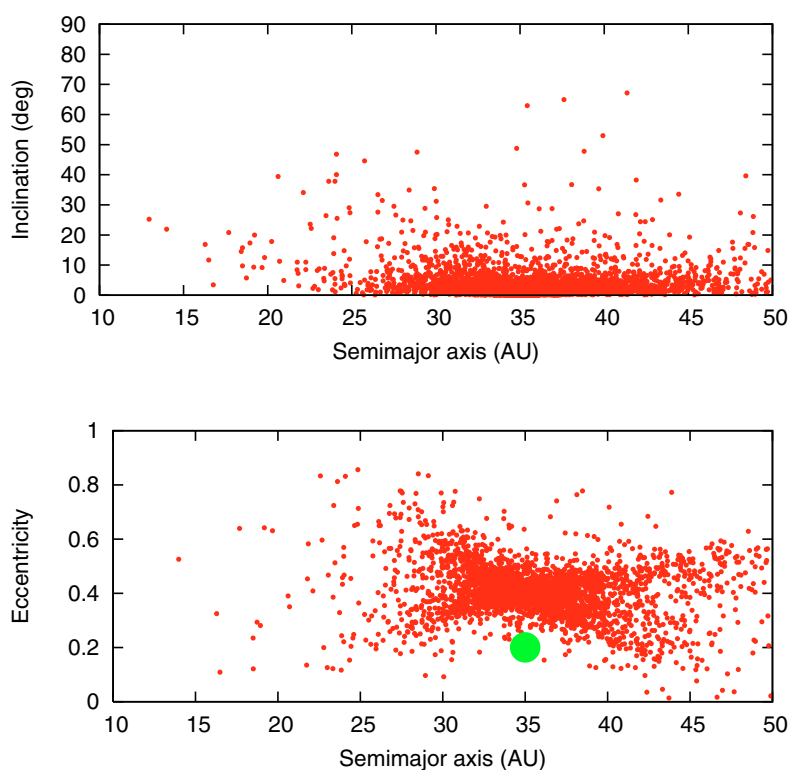

Fig. 4. Distribution of the eccentricity and inclination of the binary system after the hyperbolic stellar encounter. All the systems shown in the plot allowed the survival of at least one planet around the primary star of the system. The large dot represents the initial binary configuration.

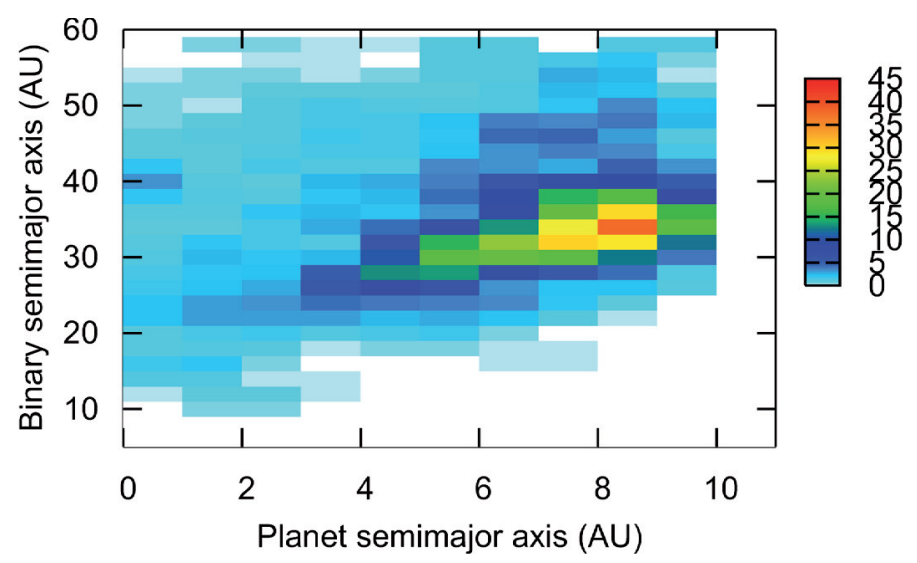

Fig. 5. Plot showing the semimajor axis $a_{\mathrm{p}}$ of the outer planetary orbit that survives the stellar encounter vs. the post-encounter semimajor axis of the companion star $a_{\mathrm{b}}$. To be compared with Fig. 3 .

on the stability of a circum-primary planetary system than the frequent close encounters between bounded stars that lead to the decay of a triple. Most of the planetary systems show orbits that are still stable up to $10 \mathrm{AU}$ when the stellar encounter is over. Of course, at later times outer orbits far from the primary may become unstable because of the long term perturbations of the companion star (Holman \& Wiegert 1999).

While the onset of instability in a triple system always leads to tighter and more eccentric binary systems, by inspecting Fig. 4 we see systems where the orbit of the binary couple is broadened and, in a few cases, even circularized by the stellar encounter. In these cases we know that planetary formation was perturbed by the companion star in the initial stages, but the dynamical environment of the planetary system became significantly quieter after the stellar encounter. There are systems where the binary companion is moved beyond some hundreds of AU from the main star. If the stellar encounter occurred after the planets formed, we might observe a broad binary system with a small planetary system around the main star. Trying to understand why 
the orbits of such a planetary system are eccentric or too close to the star without considering the possibility of a stellar encounter would lead to wrong conclusions.

\section{Conclusions}

Any attempt to model the formation and evolution of a planetary system around the component of a binary star system should account for the possibility that the binary orbit has changed with time. A dynamical event like the destabilization of an original triple system or a close stellar encounter can significantly alter the binary orbit after the formation of planets around the primary star. A system like $\gamma$ Cephei where a planet orbits the main star at about $2 \mathrm{AU}$ and is perturbed by a secondary star presently moving at about $20 \mathrm{AU}$ might have had a different dynamical configuration when the planet formed. Trying to interpret the present configuration of the system using the observed orbital and physical parameters may lead to misleading deductions on the physical properties of the protoplanetary disk that generated the planets. Prior to the binary orbit change, the disk may have been more (or less) affected by the gravitational pull of the companion star that was on a different orbit. The individual masses and orbit separations of the planets that formed in this environment might not be well simulated by introducing in the planetary formation model the present parameters of the system. Planetesimal accretion, which is the first step of terrestrial planet formation and of the core-accretion model for giant planet growth may occur differently depending on the binary orbit and companion star distance. Even gravitational instability might follow a different path if the companion star was farther away in the initial phase of the binary life.

In a few cases a stellar encounter may even cause the stripping of the companion star from the binary. The outcome would be a single star with a planetary system that formed when the star was part of a binary system and then perturbed by the gravity field of the companion star. In addition, stellar encounters may even push the orbit of the companion star out of its original plane leading to a significant mutual inclination between the planet and the companion. This is an efficient mechanism to produce planets in a Kozai resonance with the secondary star.

Acknowledgements. We thank P. Eggleton for his useful comments and suggestions while acting as referee of the paper.

\section{References}

Boss, A. 2006, ApJ, 641, 1148

dalla Stella, A., Marzari, F., Barbieri, M., Vanzani, V., \& Ortolani, S. 2005, 36th LPSC 2005, 1253

Desidera, S., \& Barbieri, M. 2007, A\&A, 462, 345

Duchene, G. 1999, A\&A, 248, 485

Eggleton, P., \& Kiseleva, L. 1995, ApJ, 455, 640

Kiseleva, L., Aarseth, S., Eggleton, P., \& de al Fuente Marcos, R. 1996, ASP Conf. Ser., 90, 433

Ghez, A. M., Neugebauer, G., \& Matthews, K. 1993, AJ, 106, 2005

Heggie, D. C. 1975, MNRAS, 283, 566

Holman, M. J., \& Wiegert, P. A. 1999, ApJ, 117, 621

Kohler, R., \& Leinert, C. 1998, A\&A, 331, 977

Kroupa, P. 1995, MNRAS, 277, 1491

Larson, R. B. 2001, in IAU Symposium, 200, ed. H. Zinnecker, \& R. D. Mathieu

Marzari, F., \& Weidenschilling, S. J. 2002, Icarus, 156, 570

Marzari, F., Weidenschilling, S. J., Barbieri, M., \& Granata, V. 2005, ApJ, 618, 502

Pollack, J. B., Hubickyj, O., Bodenheimer, P., et al. 1996, Formation of the Giant Planets by Concurrent Accretion of Solids and Gas, Icarus, 124, 62

Rasio, F. A., \& Ford, E. B. 1996, Science, 274, 954

Everhart, E. 1985 in Dynamics of comets: their origin and evolution, ed. A. Carusi, \& G. B. Valsecchi (Dordrecht: Reidel), Proc. IAU Coll., 83, 185 Reipurth, B. 2000, AJ, 120, 3177

Reipurth, B., \& Zinnecker, H. 1993, A\&A, 278, 81

Thébault, P., Marzari, F., Scholl, H., Turrini, D., \& Barbieri, M. 2004, Planetary formation in the $\gamma$ Cephei system, A\&A, 427, 1097

Thébault, P., Marzari, F., \& Scholl, H. 2006, Relative velocities among accreting planetesimals in binary systems: The circumprimary case, Icarus, 183, 193

Weidenschilling, S. J., \& Marzari, F., 1996, Nature, 384, 619 